Memorias del VII Encuentro Nacional de Experiencias en la Enseñanza de la Biologia y la Educación Ambiental y II Congreso Nacional de Investigación en la Enseñanza de La Biología

\title{
ABONANDO ANDO EN COMPETENCIA Un reto eficiente y eficaz que hay que vivenciar y disfrutar
}

\author{
ANDO paying COMPETING \\ An efficient and effective challenge to be experiencing and enjoying
}

\section{Por: Carmen Cantero $\mathrm{E}^{1}$}

\section{Resumen}

Con este proyecto se ha logrado transformar los residuos orgánicos que se producen en la institución en Compost, el cual es un producto orgánico rico en nutrientes, que constituyen un excelente abono. De igual forma, el estudiante toma conciencia de la necesidad de mantener un ambiente limpio, lo cual se proyecta a los hogares por medio de campañas culturales que van comprometiendo a su familia y la comunidad donde habitan, con esto estimula la capacidad para crear, transformar y desarrollar habilidades que generan cambios en el contexto para el mejoramiento personal y colectivo; además el estudiante comprende que con la transformación de los residuos sólidos se pueden desarrollar estándares curriculares desde las diversas áreas.

Se impulsa la cultura del emprendimiento y el desarrollo de competencias básicas, ciudadanas, investigativas y laborales mediante la estrategia del desarrollo de Proyectos Pedagógicos Productivos (PPP) que permiten mejorar los resultados académicos, dentro del marco del modelo holístico que se maneja en la institución, orientado hacia la solución de problemas mediante el método IDEAL, fundamentado en estándares y competencias. (Yus Ramos, 2001).

Se asume la metodología problemática, de aprendizaje significativo, autónomo, cooperativo, experiencial y conceptual de la enseñanza; se orienta por las técnicas del trabajo en equipo, investigación en grupo y cooperación guiada que privilegian la construcción del conocimiento, el aprendizaje en equipo y el autoaprendizaje.

Además es una herramienta metodológica que permite solucionar los estándares curriculares, tomando como base el proceso de enseñanza

\footnotetext{
${ }^{1}$ Especialista e Recreación Ecológica, Informática Educativa. Universidad los Libertadores; Universidad del Sinú.
} 
Memorias del VII Encuentro Nacional de Experiencias en la Enseñanza de la Biología y la Educación Ambiental y II Congreso Nacional de Investigación en la Enseñanza de la Biología

aprendizaje para integrar EL SABER, EL HACER Y EL SER.

Palabras clave: Investigación, ambiente, aprendizaje, innovación, competencias, abono, cultura ambiental, emprendimiento, compost.

\section{Abstract}

With the implementation of this project, the organic waste produced in the institution will be transformed into Compost, which is a nutrient-rich organic product that is an excellent fertilizer. furthermore, students become aware of the need to maintain a clean environment, which is projected to households through cultural campaigns that commit their family and the community where they live, this stimulates the ability to create, transform and develop skills that lead to changes in the context for personal and collective improvement, besides, students understand that with the transformation of solid waste can be developed curriculum standards from various areas.

It promotes the culture of entrepreneurship and the development of basic skills, citizenship, labor and investigative through the development of Productive Pedagogical Projects (PPP) that improve academic results, within the framework of the holistic model that is handled in the institution, oriented towards problem solving by IDEAL method, based on standards and competencies. (Yus Ramos, 2001); It is assumed problematic methodology, meaningful learning, autonomous, cooperative, experiential and conceptual teaching; it is guided through teamwork techniques, research group and guided cooperation that favors the construction of knowledge, team learning and selflearning.

Additionally, it is a methodological tool that helps solve curriculum standards, based on the teaching-learning process to integrate KNOWING, KNOW BEING and KNOW DOING.

Keywords: research, environment, learning, innovation, competencies, fertilizer, environmental culture, entrepreneurship, compost.

\section{Introducción}

La Institución Educativa INEM “Lorenzo María Lleras", está ubicada en ciudad de Montería - Córdoba, bordeada por el Rio Sinú, formando un valle donde crecen y se nutren una diversidad de plantas. La biodiversidad florida permea 
Memorias del VII Encuentro Nacional de Experiencias en la Enseñanza de la Biologia y la Educación Ambiental y II Congreso Nacional de Investigación en la Enseñanza de La Biología

el ambiente escolar, ofreciendo riquezas naturales utilizadas por los estudiantes para desarrollar proyectos ambientales escolares, con el fin de proporcionarle beneficio a la comunidad educativa y mantener el equilibrio de la naturaleza.

Contamos con una infraestructura muy amplia y para el aseo colaboran tres personas de servicios generales, las cuales son insuficientes para mantener un medio ambiente agradable libre de enfermedades infectocontagiosas que deterioran la salud de la comunidad. Es de anotar que existe falta de cultura ambiental y por esto al transitar por sus alrededores es fácil encontrar acumulación de residuos sólidos esparcidos por todos lados, que al no ser recogidos a tiempo ocasionan descomposiciones produciendo cierto grado de contaminación ambiental.

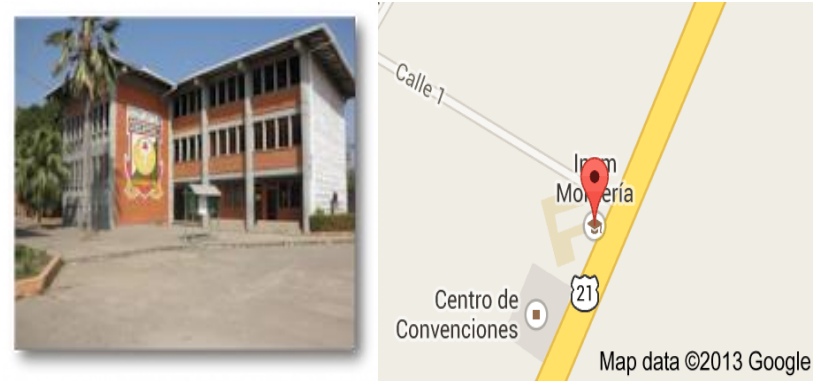

Figura 1. INEM de Montería. Fuente: Google maps.

La administración de la Institución no invierte lo necesario en el mantenimiento de jardinerías y zonas verdes, ni existe una política educativa que permitiera incluir en el currículo la educación ambiental, lo que ocasiona que solamente se realicen algunas actividades puntuales sin seguimiento alguno; sin embargo, a partir de una evaluación de dichas acciones, se detectó una serie de factores irregulares que inciden sobre el medio ambiente, surgiendo malos hábitos de los educandos en relación con su conservación. A esto se suma un manejo inadecuado de las campañas educativas ambientales, la carencia de políticas para una cultura ambiental, la falta de apoyo y de continuidad a las iniciativas individuales para adquirir esa cultura, en fin la negligencia y la falta de compromiso administrativo para mejorar el Medio Ambiente; en general, la apatía del resto de la comunidad no permitía consolidar un sentido de pertenencia.

Fue entonces, cuando nació la idea de aprovechar los residuos sólidos representados en materia orgánica, que es la componente esencial para la producción de abono orgánico, a partir de los residuos vegetales que se producen en el restaurante, jardines y zonas libres del INEM. 
Memorias del VII Encuentro Nacional de Experiencias en la Enseñanza de la Biologia y la Educación Ambiental y II Congreso Nacional de Investigación en la Enseñanza de La Biología

Todo esto atrae la búsqueda de estrategias pedagógica que se puedan implementar y que permita motivar a estudiantes, a través de un ambiente nuevo de aprendizaje para formar competencias básicas, ciudadanas, laborales e investigativas, dentro de la malla curricular de cada área. En palabras de Edgar Morin, "se pasa del currículo vicioso al currículo virtuoso, es decir, al currículo innovador, la misma vuelta del quehacer cotidiano, pero cada vez diferente y enriquecida".

"El quehacer cotidiano de maestros y el estudiante se encuentra una de las fuentes auténticas para la construcción de saber pedagógico, contenido como aquel que contextualiza es producto y da sentido al oficio de maestro". (De Tezano 1998), tomando este referente, se plantea el objetivo principal, cual es propiciar la estrategia de producción de abonos orgánicos, que permita el desarrollo en competencias que admitan incorporar la dimensión ambiental a los planes de estudio de la institución, articulando la teoría con la práctica, desarrollando acciones que contribuyan a la conservación, protección y preservación de los recursos naturales y formando integralmente para elevar la calidad de vida de los miembros de la comunidad educativa y los habitantes del entorno.

\section{Materiales y métodos}

Consideramos que la metodología más ajustada a este trabajo se fundamenta en la Investigación - Acción - Participación (IAP) ya que ésta permite involucrar a todos los actores en el problema los cuales son: estudiantes, trabajadores, directivos, profesores, padres de familia y vendedores externos, para que mediante el desarrollo de un proyecto tomen conciencia y se comprometan a asumir el estudio de su propia realidad ambiental para transformarla en procura de una mejor calidad de vida.

Esta perspectiva investigativo busca que los participantes en el proceso se reconozcan como sujetos sociales que promuevan espacios que posibiliten su encuentro, tales como: mesas de trabajo, entrevistas, encuestas, mapas sociales, reuniones o plenarias con grupos grandes o pequeños de donde debe salir un diagnóstico del problema trabajado.

Para solucionar el problema diagnosticado los estudiantes y comunidad en general participan activamente, recogiendo residuos de frutas que se producen en la casa y en nuestro entorno, el estiércol de ganado que se produce en la granja San Antonio de Berasategui y los residuos orgánicos que producen los vegetales para luego realizar el montaje de las compostera poniendo en 
Memorias del VII Encuentro Nacional de Experiencias en la Enseñanza de la Biología y la Educación Ambiental y II Congreso Nacional de Investigación en la Enseñanza de La Biología

práctica el desarrollo de competencias básicas, ciudadanas, investigativas y laborales, planteadas por el Ministerio de Educación Nacional.

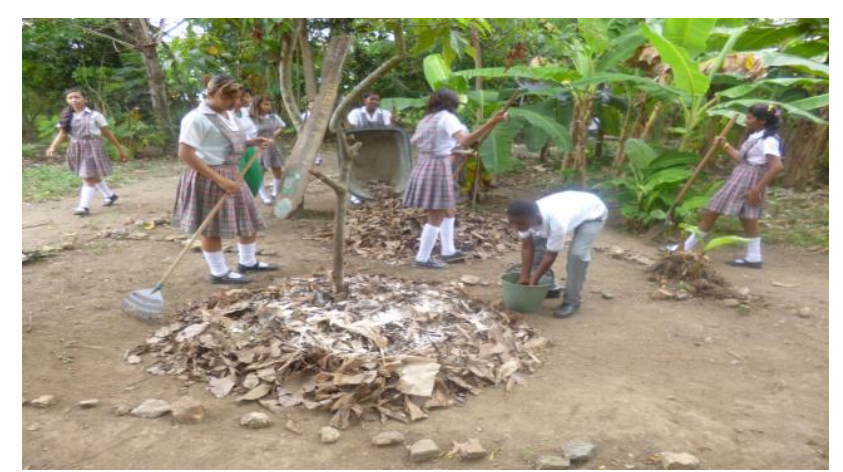

Figura 3. Realización a compostera. Fuente: Grupo de investigación.

Se trabaja en aprendizajes basados en el enfoque problémico: Se utiliza el método IDEAL:

I: Identificación del problema.

D: Descripción del problema.

E: Estrategias para resolver el problema.

A: Acciones organizadas o sistematizadas para solucionar el problema.

L: El logro final o solución del problema.

Todo esto fundamentado en estándares y competencias.

Los estándares se evalúan por actividades y competencias. Las actividades son basadas en procesos del saber, ser y hacer y las competencias por el desempeño.

Las competencias se evalúan a través del desarrollo de competencias básicas: Cuando demuestran en el hacer que existe una apropiación de una conceptualización, Ciudadana: Cuando se muestra como un ser social, solidario, con amor a la naturaleza. Líder, creativo, emprendedor y trabaja en equipo.

Para la ejecución de las actividades contamos con patios muy amplios, un aula ambiental y el Centro Experimental de Educación Ambiental "Las Iguanas", que es utilizado como laboratorio de aprendizaje; es allí donde se montan las unidades de procesamiento de residuos para obtener abono.

En el trabajo de campo, empiezan a desarrollar las competencias ciudadanas y básicas, plantadas en las cartillas del MEN integrándolas con las investigativas, de acuerdo a lo formulado por "José Federman", haciendo el montaje respectivo de las aboneras. 
Memorias del VII Encuentro Nacional de Experiencias en la Enseñanza de la Biología y la Educación Ambiental y II Congreso Nacional de Investigación en la Enseñanza de La Biología

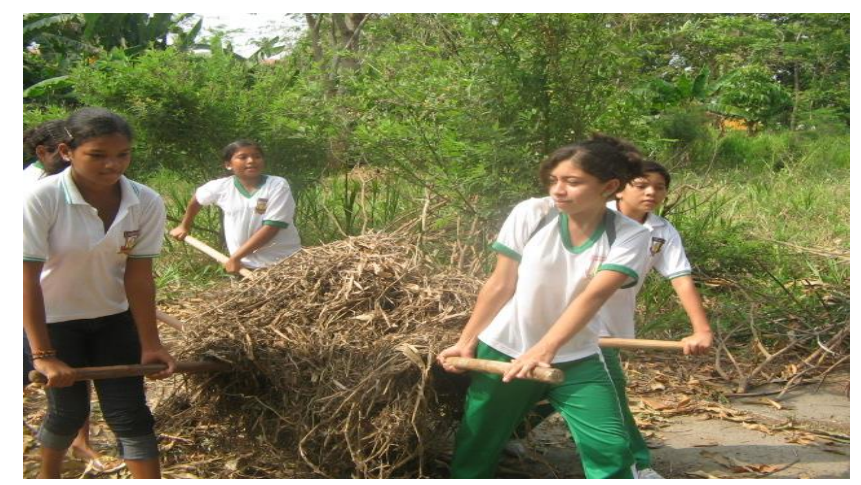

Figura 2. Recolección de materiales. Fuente: Grupo de investigación.

En esta acción cada grupo tiene una función: unos se dedican a organizar y transportar la materia orgánica, otros preparan el estiércol, unos inician el montaje de las compostera, los demás consiguen el agua, la ceniza o la cal, otros manejan y cuidan las herramientas.

En cada salida los estudiantes deben responder por diversas actividades, aplicadas a las áreas de Español, Matemática, Sociales, Inglés, Ciencias Naturales, Química, y Artística. "Qué rico alcanzar cinco o seis logros", dicen los estudiantes.

Para recoger los frutos del trabajo el equipo debe echar para adelante, por eso toca tirar responsabilidad, ya que cada 15 o 21 días debemos regresar al laboratorio a realizar seguimiento, es necesario voltear y mezclar los materiales. En este proceso se inicia la parte más bonita, debido a que los educandos deben clasificar microorganismos, estudiar flujo de energía, medir temperaturas, acelerar la descomposición de los microorganismos, pesar y observar, con el fin de poner en práctica las competencias relacionadas con los estándares curricular, desplegando en el aula de clases, utilizando la interdisciplinariedad con las demás aéreas. 
Memorias del VII Encuentro Nacional de Experiencias en la Enseñanza de la Biologia y la Educación Ambiental y II Congreso Nacional de Investigación en la Enseñanza de La Biología

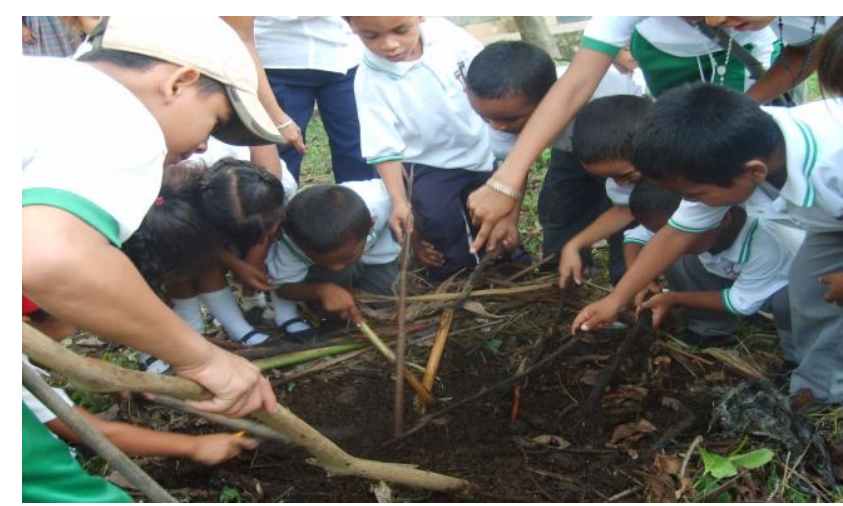

Figura 4. Investigando. Fuente: Grupo de investigación.

Después del primer esfuerzo, como si fuera poco, pasado noventa días obtenemos un producto de buena calidad, lo que permite trabajar y apoyar otros proyectos, tales como: mi vivero escolar, plantas medicinales, pan coger, ornamentación y embellecimiento; de ese modo se amplía el abanico del desarrollo de competencias básicas en el área de Ciencias Naturales.

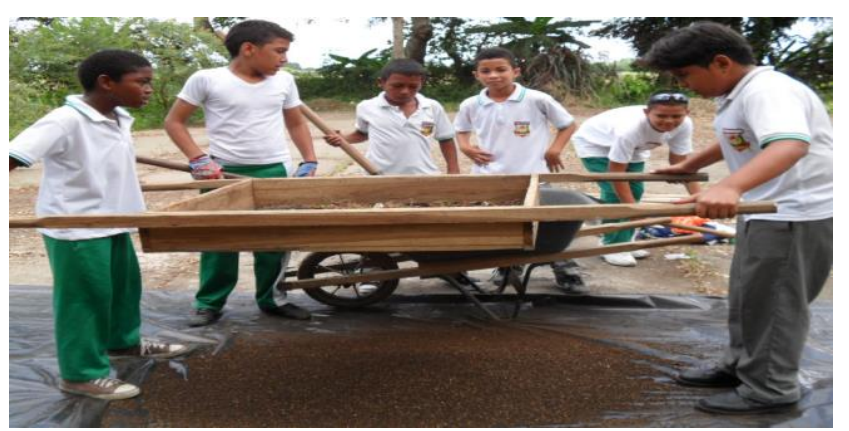

Figura 5. Tamizado. Fuente: Grupo de investigación

En esa dinámica de trabajo y estudio se aborda lo concerniente a la capacidad organizacional cuando llega la hora de mostrar el trabajo, iqué satisfacción!, ahora deben tamizar, secar y llevar la producción del abono al Aula Ambiental, donde aunando esfuerzos se empaca, se rotula y se almacena en la Tienda Ecológica para ser distribuido a los clientes internos y externo. 
Memorias del VII Encuentro Nacional de Experiencias en la Enseñanza de la Biologia y la Educación Ambiental y II Congreso Nacional de Investigación en la Enseñanza de La Biología

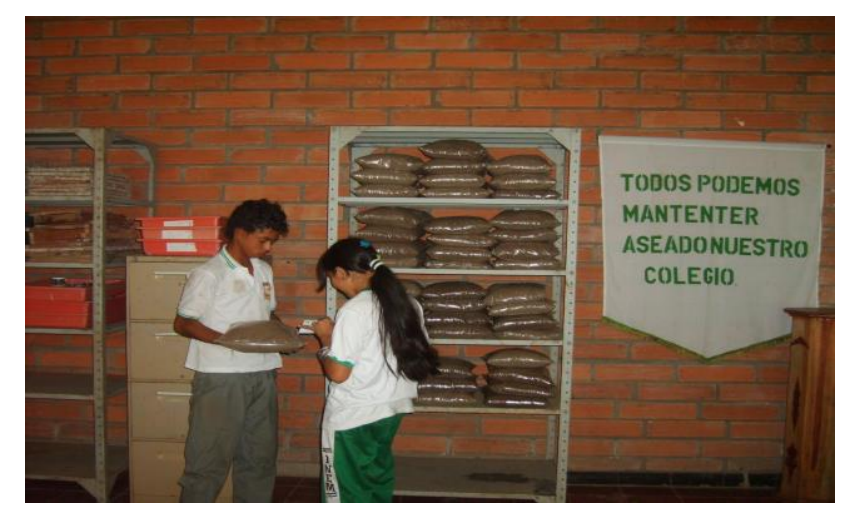

Figura 6. Empaque y rotulación. Fuente: Grupo de investigación

Pero esto no queda entre nosotros, se siguen conquistando metas, los estudiantes son felices mostrando los resultados del trabajo que ellos realizan, por esta razón participan en todos los eventos ambientales realizados por la CVS, la Secretaría de Educación Municipal y Departamental y las Instituciones públicas o privadas; finalmente, se goza con ese resultado orientado por el desarrollo de las competencias y las derivadas del conocimiento y el aprendizaje.

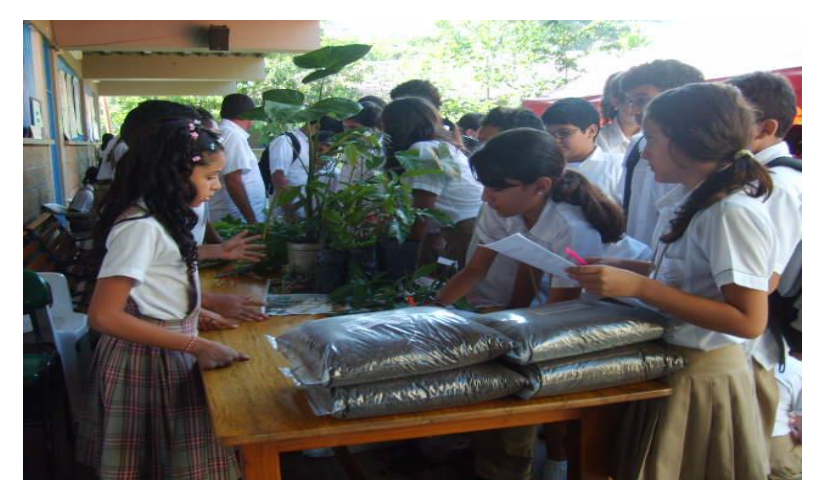

Figura 7. Participación en eventos. Fuente: Grupo de investigación

\section{Resultados y discusión}

En mi condición de docente puedo dar fe de que el trabajo por proyectos va más allá de generar interés en los estudiantes. Estos alientan una indagación activa y un pensamiento de orden superior. Las habilidades de los estudiantes en la adquisición de nuevos entendimientos son acentuados cuando ellos son vinculados con acciones significativas en la solución de problemas y se les ayuda a comprender el por qué, cuándo y cómo esos hechos y destrezas son relevantes. Además, te ayuda a mejorar profesionalmente, aumenta la colaboración entre colegas, se tiene mayor oportunidad de consolidar las 
Memorias del VII Encuentro Nacional de Experiencias en la Enseñanza de la Biología y la Educación Ambiental y II Congreso Nacional de Investigación en la Enseñanza de la Biología

relaciones con los estudiantes y a encontrar un modelo que se logre acomodar a la diversidad de los aprendices.

Es emocionante observar cómo el aprendiz exploran de forma espontánea y creativa el ambiente circundante, dejando manar "ese yo explorador". En este sentido, la investigación se convierte en una actividad imprescindible a lado de los alumnos, pues, la escuela está llamada a convertirse en el escenario permanente de interacción y transformación social, de tal forma que hay la necesidad de liderar la realización y ejecución de proyectos productivos pedagógicos productivos que fomenten el emprendimiento, la investigación y permitan la solución de algunos problemas de la realidad escolar.

Con la ejecución de experiencias significativas se fortalece el compromiso, la integración, la sostenibilidad y la productividad en beneficio de una comunidad con propósitos colectivos, que logre implementar la transversalización como una carta de navegación y que trascienda los muros extracurriculares de las comunidades del entorno. El reto es lograr el empoderamiento de estas estrategias en el conglomerado de docentes interesados en fortalecer su quehacer e interesados en compartir enseñanzas y aprendizajes transformadores.

Hoy se afirmar que los estudiantes han mejorado sus resultados académicos, sociales y personales, ya que se han empoderado de una metodología didáctica y creativa, que les permite mostrar cambios, como:

- Apropiados de las competencias básicas y ciudadanas, mejorando su rendimiento académico, comportamiento con vivencial y capacidad de trabajo.

- Estudiantes líderes, autónomos, emprendedores, con capacidad de organización, con valores cimentados y aplicando herramientas tecnológicas.

- La transversalidad les permite desarrollar competencias en diferentes áreas.

- Se evidencia, la disminución de la deserción escolar, incrementando la confianza en sí mismos y el mejoramiento de actitud hacia el aprendizaje.

- Los cambios en la disciplina general de la Institución saltan a la vista, ya que los estudiantes practican un comportamiento social adecuado, manejan su inteligencia emocional, actúan con un sentido ético y son permeables a los cambios. 
Memorias del VII Encuentro Nacional de Experiencias en la Enseñanza de la Biologia y la Educación Ambiental y II Congreso Nacional de Investigación en la Enseñanza de La Biología

- Se le está facilitando, relacionarse con el entorno productivo y fortalecer mediante la transversalidad el desarrollo curricular del núcleo común, cimentando las bases para la formación de las diferentes competencias.

- Mejores resultado en las pruebas saber de los grados nueve y once, ya que la Institución paso del nivel bajo a alto.

- Utilización de las TIC, como una herramienta pedagógica y es por esto que hoy contamos con un blog del proyecto (http://abonorganicoinem.blogspot.com) y con un blog institucional (http://inemonteria.blogspot.com/).

- La evaluación es orientada por el desarrollo de las competencias y las derivadas del conocimiento y el aprendizaje. Es permanente, formativa, centrada en el proceso y el logro de los estudiantes, integra la dimensión cualitativa sobre lo evaluado, el conocimiento logrado, el nivel de cumplimiento de las metas, mostrando los resultados en el proceso en enseñanza aprendizaje. Se dinamiza mediante la autoevaluación, Coevaluación y heteroevaluaciòn. Todo lo anterior implica la elaboración de instrumento de evaluación y recolección de evidencias de aprendizaje.

\section{Conclusiones}

- El proyecto ha permitido generar espacios pedagógicos reales que permiten el desarrollo de los procesos educativos de manera práctica y vivencial.

- Construir ambientes de aprendizaje favorables, que permitan la integración de las áreas básicas y optativas del plan de estudios y su aplicación práctica.

- Desarrollar estrategias de proyección de la institución educativa a la comunidad.

- Promover la implementación de prácticas ambientalmente sostenibles.

- Desarrollar un currículo contextualizado y de conformidad con las necesidades, vinculando a los estudiantes con experiencias de producción, transformación, comercialización, consumo y creación de empresa. 
Memorias del VII Encuentro Nacional de Experiencias en la Enseñanza de la Biología y la Educación Ambiental y II Congreso Nacional de Investigación en la Enseñanza de La Biología

- Aumentar la colaboración entre colegas, se tiene mayor oportunidad de aumentar las relaciones con los estudiantes y encontrar un modelo que logre acomodar diversos aprendices.

- Con el inicio de un nuevo año surgen nuevas investigaciones que se convierten en desafíos, lo que permite seguir alcanzando logros pedagógicos, apropiarnos del mercado emprendedor y seguir mejorando la calidad de nuestros educandos.

\section{Bibliografía}

COLOMBIA. MINISTERIO DE EDUCACIÓN NACIONAL. Aporte para la construcción de currículos pertinentes. (2006). Competencias laborales Generales Articulación de la educación con el Mundo productivo. Serie de guía No 21.

COLOMBIA. MINISTERIO DE EDUCACIÓN NACIONAL. Estándares Básicos de Competencias en Lenguaje, Matemáticas, Ciencias y Ciudadanas. (2006). Guía sobre lo que los estudiantes deben saber y saber hacer con lo que aprenden. Revolución Educativa Colombia Aprende.

COLOMBIA. MINISTERIO DE EDUCACIÓN NACIONAL. Estándares Básicos de Competencias, en Ciencias Naturales y Ciencias Sociales. Serie Guía No 7.

COLOMBIA. MINISTERIO DEL MEDIO AMBIENTE. (2002). Proyecto de Gestión Integral de Residuos Sólidos. Guía práctica de formulación

José Federmann Muñoz Giraldo, Josefina Quintero Orozco, Raúl Ancizar Munevar Molina. (2005). Como desarrollar Competencias Investigativas en educación. Tercera Edición. Cooperativa Editorial del Magisterio.

Tobasura Acuña Isaías, Sepulveda gallego luz Elena. (1997). Proyectos Ambientales Escolares. Estrategia para la formación Ambiental. Editorial Magisterio. Páginas: 33, 34,90 y 91. 